\title{
DARK MATTER BURNERS
}

\author{
IGOR V. MOSKALENKO ${ }^{1}$ \\ Hansen Experimental Physics Laboratory, Stanford University, Stanford, CA 94305 \\ AND \\ LAWRENCE L. WAI ${ }^{1}$ \\ Stanford Linear Accelerator Center, Stanford University, 2575 Sand Hill Rd, Menlo Park, CA 94025 \\ Draft version February 24, 2007
}

\begin{abstract}
We show that a star orbiting close enough to an adiabatically grown supermassive black hole (SMBH) can capture weakly interacting massive particles (WIMPs) at an extremely high rate. The stellar luminosity due to annihilation of captured WIMPs in the stellar core may be comparable to or even exceed the luminosity of the star due to thermonuclear burning. The model thus predicts the existence of unusual stars, essentially WIMP burners, in the vicinity of a SMBH. We find that the most efficient WIMP burners are stars with degenerate electron cores, e.g. white dwarfs (WDs); such WDs may have a very high surface temperature. If found, such stars would provide evidence for the existence of particle dark matter and can possibly be used to establish its density profile. On the other hand, the lack of such unusual stars may provide constraints on the WIMP density near the SMBH, as well as the WIMP-nucleus scattering and pair annihilation cross-sections.
\end{abstract}

Subject headings: black hole physics — elementary particles — radiation mechanisms: non-thermal — stars: general — stars: evolution — dark matter

\section{INTRODUCTION}

The nature of the non-baryonic dark matter, which dominates the visible matter by about $4: 1$, is perhaps the most interesting experimental challenge for contemporary particle astrophysics. A hint for a solution has been found in particle physics where the WIMPs arise naturally in supersymmetric extensions of the Standard Model (e.g., Haber \& Kane 1985), among other possibilities. The WIMP is typically defined as a stable, electrically neutral, massive particle. Assuming that non-baryonic dark matter is dominated by WIMPs, the pair annihilation cross-section is related to the observed relic density (Bergström 2000; Jungman, Kamionkowski \& Griest 1996). A pair of WIMPs can annihilate producing ordinary particles and $\gamma$-rays.

WIMPs are expected to form high density clumps according to N-body simulations of test particles with only gravitational interactions (Moore et al. 1999; Navarro, Frenk, \& White 1997). The highest density "free space" dark matter regions occur for dark matter particles captured within the gravitational potential of adiabatically grown SMBHs (Bertone \& Merritt 2005; Gnedin \& Primack 2004; Gondolo \& Silk 1999). Higher dark matter densities are possible for dark matter particles captured inside of stars or planets. Any star close enough to a SMBH can capture a large number of WIMPs during a short period of time. Annihilation of captured WIMPs may lead to considerable energy release in stellar cores thus affecting the evolution and appearance of such stars.

Such an idea has been first proposed by Salati \& Silk (1989) and further developed by Bouquet \& Salati (1989) who applied it to main-sequence stars. The model led to the conclusion of suppression of stellar core convection, thus predicting a concentration of stars in the Galactic Center masquerading as cold red giants.

An order-of-magnitude estimate of the WIMP capture

\footnotetext{
${ }^{1}$ Also Kavli Institute for Particle Astrophysics and Cosmology, Stanford
} University, Stanford, CA 94309 rates for stars of various masses and evolution stages (Moskalenko \& Wai 2006) lead us to the conclusion that WDs, fully burned stars without their own energy supply, are the most promising candidates to look for. In this paper we calculate the WIMP capture by WDs located in a high density dark matter region, and discuss their observational features. We use current limits on WIMP-nucleus interaction and WIMP annihilation cross sections, as well as recent estimates of WIMP energy density near an adiabatically grown SMBH.

\section{WIMP ACCUMULATION IN STARS}

In a steady state the WIMP capture rate $C$ is balanced by the annihilation rate (Griest \& Seckel 1987)

$$
C=A N_{\chi}^{2}
$$

where

$$
A=\frac{\left\langle\sigma_{a} v\right\rangle}{\pi^{3 / 2} r_{\chi}^{3}},
$$

$\left\langle\sigma_{a} v\right\rangle$ is the velocity averaged WIMP pair annihilation crosssection, the effective radius

$$
r_{\chi}=c\left(\frac{3 T_{c}}{2 \pi G \rho_{c} m_{\chi}}\right)^{1 / 2}
$$

is determined by matching the star core temperature $T_{c}$ with the gravitational potential energy (assuming thermal equilibrium), $c$ is the speed of light, $G$ is the gravitational constant, $\rho_{c}$ is the star core density, and $m_{\chi}$ is the WIMP mass. The total number of WIMPs captured by a star is

$$
N_{\chi}=C \tau_{e q} \tanh \left(\tau_{*} / \tau_{e q}\right),
$$

where $\tau_{*}$ is the star's age, and the equilibrium time scale is given by

$$
\tau_{e q}=(C A)^{-1 / 2} .
$$

Limits from direct detection of dark matter on the WIMPnucleon cross-section imply that only a fraction of the WIMPs 

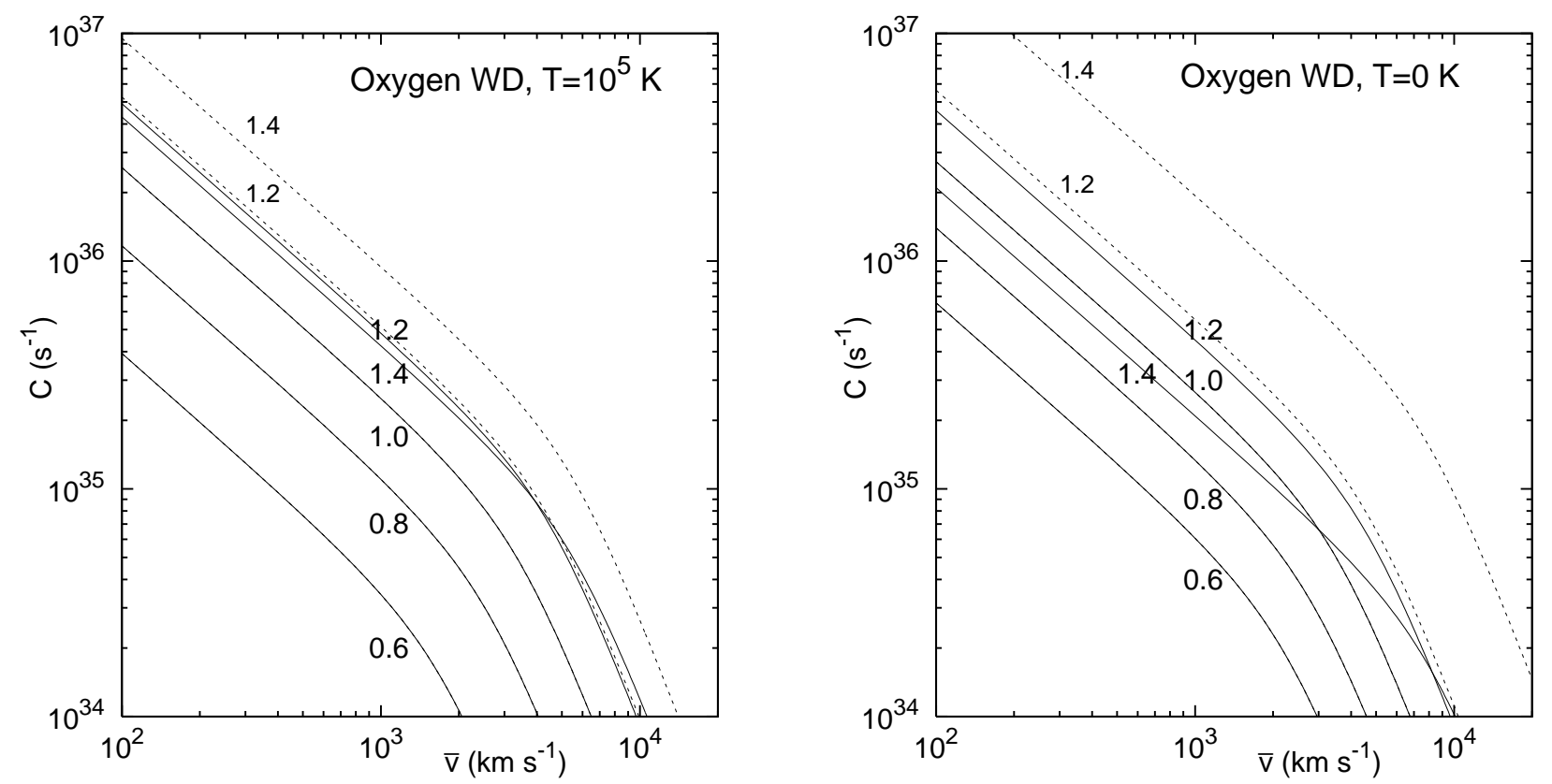

FIG. 1.-WIMP capture rate by Oxygen WDs $\left(A_{n}=16\right)$ vs. velocity dispersion for $\rho_{\chi}=\rho_{\chi}^{\max }$. The left panel shows calculations for WDs without hydrogen envelope and $T=100,000 \mathrm{~K}$ (Panei et al. 2000). Right panel corresponds to the zero-temperature approximation (Hamada \& Salpeter 1961). The solid lines show the capture rate calculated using the modified cross section (Eq. 9 ), the dashed lines are calculated for $\sigma_{0}^{\prime}=\sigma_{0}$. The labels show WD mass in $M_{\odot}$ units.

crossing the star will scatter and be captured. The capture rate for a Maxwellian WIMP velocity distribution (in the observer's frame) by a star moving with an arbitrary velocity $v_{*}$ relative to the observer is given by (Gould 1987):

$$
C=4 \pi \int_{0}^{R_{*}} d r r^{2} \frac{d C(r)}{d V}
$$

where

$$
\begin{aligned}
\frac{d C(r)}{d V} & =\left(\frac{6}{\pi}\right)^{1 / 2} \sigma_{0} A_{n}^{4} \frac{\rho_{*}}{M_{n}} \frac{\rho_{\chi}}{m_{\chi}} \frac{v^{2}(r)}{\bar{v}^{2}} \frac{\bar{v}}{2 \eta A^{2}} \\
& \times\left\{\left(A_{+} A_{-}-\frac{1}{2}\right)\left[\chi(-\eta, \eta)-\chi\left(A_{-}, A_{+}\right)\right]\right. \\
& \left.+\frac{1}{2} A_{+} e^{-A_{-}^{2}}-\frac{1}{2} A_{-} e^{-A_{+}^{2}}-\eta e^{-\eta^{2}}\right\} \\
A^{2} & =\frac{3 v^{2}(r) \mu}{2 \bar{v}^{2} \mu_{-}^{2}} \\
A_{ \pm} & =A \pm \eta, \\
\eta & =\frac{3 v_{*}^{2}}{2 \bar{v}^{2}}, \\
\chi(a, b) & =\int_{a}^{b} d y e^{-y^{2}}=\frac{\sqrt{\pi}}{2}[\operatorname{erf}(b)-\operatorname{erf}(a)]
\end{aligned}
$$

$\rho_{\chi}$ is the ambient WIMP energy density, $A_{n}$ is the atomic number of the star's nuclei, $M_{n}$ is the nucleus mass, $\bar{v}$ is the WIMP velocity dispersion, and $\mu=m_{\chi} / M_{n}, \mu_{-}=(\mu-$ $1) / 2$. The escape velocity at a given radius $r$ inside of a star is given by

$$
v(r)=\left[2 G \int_{V_{*}} d V \frac{\rho_{*}(r)}{r}\right]^{1 / 2}=\left[\frac{G M_{*}}{R_{*}}\left(3-\frac{r^{2}}{R_{*}^{2}}\right)\right]^{1 / 2},
$$

where we assumed the same mass density $\rho_{*}=M_{*} / V_{*}$ and the same chemical composition over the entire scattering volume $V_{*}$. This is a reasonable assumption for a degenerate electron core. Near a SMBH, where orbital motion around a single mass dominates, the test particle (WIMP or star) velocities are Keplerian $v_{*}=\bar{v}$; in this case $\eta=3 / 2$, although the exact value does not significantly change the result. The value of the spin-independent WIMP-nucleon scattering cross-section $\sigma_{0}$ is limited by direct detection experiments, i.e. less than $10^{-43} \mathrm{~cm}^{2}$ (CDMS 2006). If the star is composed of nuclei with atomic number $A_{n}$, the cross section increases by a coherent factor of $A_{n}^{4}$.

If a WD is heavy $\left(M \gtrsim M_{\odot}\right)$ and/or $A_{n} \gg 1$, almost all WIMPs crossing the star will be captured. In this case, the WIMP capture rate is determined by the geometrical limit $\pi R_{*}^{2}$ rather than the total interaction cross section $\sigma_{0} A_{n}^{4} M_{*} / M_{n}$. We thus use a modified interaction cross section $\sigma_{0}^{\prime}$ defined as

$$
\sigma_{0}^{\prime} A_{n}^{4} \frac{M_{*}}{M_{n}}=\min \left(\sigma_{0} A_{n}^{4} \frac{M_{*}}{M_{n}}, \pi R_{*}^{2}\right) .
$$

Figure 1 shows the capture rate by Oxygen WDs $\left(A_{n}=16\right)$ vs. WIMP velocity dispersion for several masses of WDs, assuming Keplerian orbits around the SMBH, $m_{\chi}=100$ $\mathrm{GeV}, \sigma_{0}=10^{-43} \mathrm{~cm}^{2}, \rho_{\chi} \sim \rho_{\chi}^{\max } \sim m_{\chi} /\left(\left\langle\sigma_{a} v\right\rangle \tau_{\mathrm{bh}}\right) \sim$ $10^{10} \mathrm{GeV} \mathrm{cm}^{-3}$ which corresponds to the maximal central particle dark matter density allowed by the age of the $\mathrm{SMBH} \tau_{\mathrm{bh}} \sim 10 \mathrm{Gyr}$ and our selected value $\left\langle\sigma_{a} v\right\rangle=$ $3 \times 10^{-26} \mathrm{~cm}^{3} \mathrm{~s}^{-1}$ (Bertone \& Merritt 2005; Gondolo \& Silk 1999). The left panel corresponds to WD effective temperature of $T=100,000 \mathrm{~K}$ (without an envelope), where the masses and radii used in the calculation are (Fig. 5 in Panei et al. 2000): $M_{*} / M_{\odot}=0.6,0.8,1.0,1.2,1.4$, and $R_{*} / R_{\odot}=0.02,0.012,0.0085,0.006,0.0045$, correspondingly. The right panel corresponds to the zero-temperature approximation (Hamada \& Salpeter 1961), where the massradius relation has been obtained by fitting the numerical results for Carbon WDs with a function $R_{*} / R_{\odot}=0.94-$ $0.67 \tan \left(1.49\left[M_{*} / M_{\odot}-0.85\right]\right)$ in the interval $M_{*} / M_{\odot}=$ 

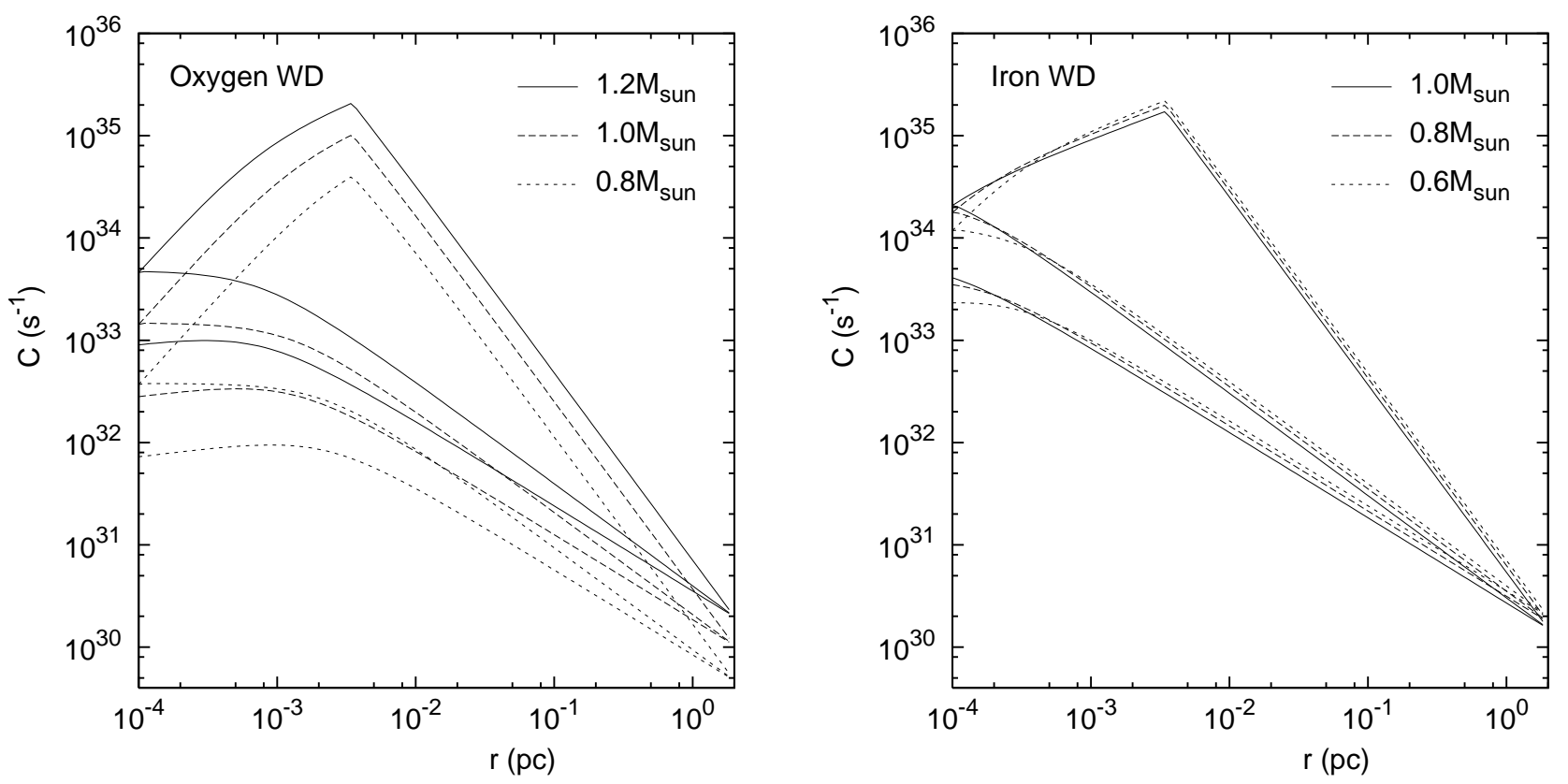

FIG. 2.- Capture rate vs. distance from the central black hole for Oxygen (left) and Iron (right) WDs of $T=100,000 \mathrm{~K}$ assuming Keplerian velocities for WIMPs and the WD. The WIMP mass density is normalized as $\rho_{\chi}(2 \mathrm{pc})=100 M_{\odot} \mathrm{pc}^{-3}$. For the central spike we assume a power-law profile with the indices (top to bottom): $7 / 3,3 / 2,4 / 3$, where the maximal possible WIMP mass density $\rho_{\chi}^{\max }$ is given in the text. The mass-radius relation is taken from Panei et al. (2000).

$0.15-1.4$. The solid lines show the capture rate calculated using the modified interaction cross section (eq. [9]), and the dashed lines are calculated for $\sigma_{0}^{\prime}=\sigma_{0}$. In the case of Oxygen WDs the geometrical limit is reached for $M_{*} \sim 1.2 M_{\odot}$; the larger mass WDs have smaller radii and therefore smaller capture rates. For $\bar{v} \lesssim 10^{3} \mathrm{~km} \mathrm{~s}^{-1}$ our geometrical limit calculations agree well with the results of Bottino et al. (2002), their equation (26). For $\bar{v} \gtrsim 10^{3} \mathrm{~km} \mathrm{~s}^{-1}$ the Bottino et al. (2002) formula, derived under the assumption that each WIMP crossing the star is captured, gives a systematically larger capture rate, up to a factor of 10 for $10^{4} \mathrm{~km} \mathrm{~s}^{-1}$. This can be treated as an upper limit, whereas our approximation to the geometrically limited case can be considered as a lower limit.

It can be seen (Figure 1) that cooler WDs have a capture rate (eqs. [6], [7]) larger than hot ones of the same mass because of the larger escape velocity (eq. [8]). The latter is the result of a smaller radius $R_{*}$ and consequently stronger gravity. This effect may be explained in terms of the "focusing factor" (Gould 1987) or simply because WIMPs can be captured from the larger volume of the Maxwellian velocity phase space. A larger capture rate by a cooler WD will lead to accelerated heating until the WD radius increases due to increased temperature. A hotter WD will be less efficient for WIMP capture and cool down. This mechanism will thus lead to fast self-regulation of the WD temperature and capture rate.

The capture rate for a different $\left(A_{n}\right)$ composition WD can be estimated from scaling the Oxygen WD curves by a factor of $\left(A_{n} / 16\right)^{3}$, e.g., the curves for a Carbon WD can be obtained from scaling the Oxygen WD curves down by a factor of $(3 / 4)^{3}$. WDs with heavier nuclei (up to iron) may exist (Panei et al. 2000); in this case, the capture rate is restricted mostly by the geometrical limit.

Figure 2 shows the capture rate for Oxygen (left panel) and Iron (right panel) WDs vs. distance from the central black hole with $M_{\mathrm{bh}}=3.7 \times 10^{6} M_{\odot}$ (Ghez et al. 2005); this includes effects of the radial dependence of the WIMP velocity dispersion and the WD orbital (Keplerian) velocity. Following Gnedin \& Primack (2004) and Bertone \& Merritt (2005), the WIMP mass density is normalized as $\rho_{\chi}(2 \mathrm{pc})=$ $100 M_{\odot} \mathrm{pc}^{-3}$. For the central spike we assume a powerlaw profile with indices (top to bottom): 7/3, 3/2, 4/3; these profiles are predicted for different scenarios of the black hole growth, adiabatic (Ullio et al. 2001), quasi-equilibrium (Gnedin \& Primack 2004), and instanteneous (Ullio et al. 2001), correspondingly. Here we use the same estimate for $\rho_{\chi}^{\max }$ as for Figure 1] The mass-radius relation for Oxygen and Iron WDs of $T=100,000 \mathrm{~K}$ is taken from Panei et al. (2000).

The capture rate scales linearly with the WIMP density, so that the largest capture rate is reached with the adiabatic profile. The capture rate increases toward the SMBH until the maximal WIMP mass density $\rho_{\chi}^{\max }$ is reached; then the capture rate decreases due to increases in the WIMP velocity dispersion and the orbital velocity of the star. For the quasiequilibrium profile, $\rho_{\chi}^{\max }$ is reached only at $\sim 10^{-4} \mathrm{pc}$, while the instanteneous profile is even flatter.

As can be seen from Figure 2 and a simple inspection of the capture rate formulae, in the geometrically limited case (eq. 9]) the capture rate becomes essentially independent of the WIMP-nucleon scattering cross-section and degenerate core parameters. Observationally, the brightest WIMP burners may be the geometrically limited ones. The main uncertainty in the geometrically limited capture rate is the dark matter density; thus it may be possible to perform largely "model independent" measurements of the dark matter density profile by measuring the luminosity of different WIMP burners orbiting within a particular dark matter spike.

A smaller annihilation cross section $\left\langle\sigma_{a} v\right\rangle<3 \times 10^{-26}$ $\mathrm{cm}^{3} \mathrm{~s}^{-1}$ would allow for larger ambient WIMP densities near the SMBH. This would lead to a larger capture rate and con- 
sequently larger burning rate at the innermost radii. The energy release due to WIMP annihilation in the stellar core is $L_{\chi} \sim 0.16 C\left(m_{\chi} / 100 \mathrm{GeV}\right) \mathrm{erg} \mathrm{s}^{-1}$ which is actually independent of the WIMP mass $m_{\chi}$.

\section{DISCUSSION}

Where does the energy released during the WIMP annihilation go? Table 1 in Moskalenko \& Wai (2006) shows that the effective radius of the thermal distribution of WIMPs in the stellar core is much smaller than the radius of the star $r_{\chi} \ll R_{*}$, therefore, the products of WIMP annihilation cannot propagate to the stellar surface and are converted into thermal energy and neutrino emission. A WD, a star without its own energy supply consisting of Carbon and Oxygen, may emit up to $L_{\chi} \sim 3 \times 10^{34} \mathrm{erg} \mathrm{s}^{-1}$, i.e. $\sim 10$ times the luminosity of the sun, burning WIMPs only and this energy source will last forever! (Note that this estimate is based on our approximation to the geometrically limited case and larger luminosities are possible even for the given set of parameters.) At such a luminosity, the surface temperature of the WD would be close to $\sim 140,000 \mathrm{~K}$, assuming $M_{*}=1.2 M_{\odot}$, $R_{*}=0.006 R_{\odot}$. The maximum of the black body emission falls into the UV band making such stars strong thermal UV emitters concentrated in the inner $\sim 0.01 \mathrm{pc}$. A smaller annihilation cross section $\left\langle\sigma_{a} v\right\rangle<3 \times 10^{-26} \mathrm{~cm}^{3} \mathrm{~s}^{-1}$ and/or larger WIMP density normalization $\rho_{\chi}(2 \mathrm{pc})>100 M_{\odot} \mathrm{pc}^{-3}$ would allow for a larger ambient WIMP density near the $\mathrm{SMBH}$, thus increasing the capture and burning rates further.

The energy transport in the interiors of WDs is dominated by degenerate electrons and is very efficient (see Hansen 2004, for a recent review); therefore, the large number of captured WIMPs and their annihilation in the core would not change the internal structure of WDs. A recently published catalog of spectroscopically confirmed WDs from the Sloan Digital Sky Survey (SDSS) (Eisenstein et al. 2006) contains several hot WDs with surface temperature in the range of $100,000 \mathrm{~K}$, thus providing observational evidence that high temperature does not change the appearance of WDs. A bare WD with an effective temperature as high as 170,000-200,000 $\mathrm{K}$ has also been observed (H1504+65, Werner \& Wolff 1999).

The number of very hot WDs in the SDSS catalog is small, just a handful out of 9316. This means that observation of a concentration of very hot WDs at the Galactic Center would be extremely unlikely unless they are "dark matter burners." The spectra of confirmed hot WDs can serve as templates for spectroscopic analysis of WDs at the Galactic Center where only a limited part of the near-IR band can be used. An inde- pendent determination of the $M_{*} / R_{*}$ ratio is possible using the gravitational redshift that has to be equivalent to a radial velocity of about $50 \mathrm{~km} \mathrm{~s}^{-1}$ (Greenstein \& Trimble 1967).

A bare WD with a highly eccentric orbit around the central black hole may exhibit variations in brightness correlated with the orbital phase (Figure 2). To have this working, the orbital period should exceed the equilibrium time scale $\tau_{e q}$ (eq. [5]). Carbon burning stars have $\tau_{e a} \sim 10 \mathrm{yr}$, and it is even shorter $\sim 0.5 \mathrm{yr}$ in case of a WD (Moskalenko \& Wai 2006). If a WD appears in a high-density WIMP region, the WIMP density in its material would quickly reach equilibrium; thus, the surrounding WIMP density variation as the WD orbits would result in variations of brightness. This makes bare WDs ideal objects to test the WIMP density in the environment in which they are orbiting. Since $L_{\chi} \propto \rho_{\chi}$, a population of WDs, bare or with envelopes, located at different distances from the SMBH would exhibit a luminosity correlated with the radial WIMP density profile. Geometrically limited WIMP burners have the highest luminosities and therefore will be the easiest to observe. Their luminosity is largely independent of WIMP-nucleon scattering cross-section, WIMP pair annihilation cross-section, and degenerate core parameters.

Advances in near-IR instrumentation have made possible observations of stars in the inner parsec of the Galaxy (Genzel et al. 2000; Ghez et al. 2003, 2005). The apparent K-band brightness of these stars is 14-17 mag. The observed absorption line widths imply high temperatures and lead to a "paradox of youth:" apparently young stars in the region whose current conditions seem to be inhospitable to star formation. One of the possibilities is that they are old stars masquerading as youths. Assuming a central spike with index 7/3, the K-band brightness for Oxygen WDs with $T \sim 100,000 \mathrm{~K}$ and $R_{*} / R_{\odot} \sim 0.01$ is about $22-23$ mag not including extinction, which may be as large as $3.3 \mathrm{mag}$ (Rieke et al. 1989). It is, therefore, unlikely that the currently observed stars in the K-band are WDs burning WIMPs; however, stars with degenerate electron cores plus envelopes cannot be ruled out.

We thank R. Blandford, J. Edsjö, J. Faulkner, S. Kahn, J. Primack, and R. Romani for interesting discussions and the anonymous referee for useful comments. L. L. W. would like to thank S. Nagataki for interesting discussions on massive stars. I. V. M. acknowledges partial support from NASA Astronomy and Physics Research and Analysis Program (APRA) grant. A part of this work was done at Stanford Linear Accelerator Center, Stanford University, and supported by Department of Energy contract DE-AC03-768SF00515.

\section{REFERENCES}

Bergström, L. 2000, Rept. Prog. Phys., 63, 793

Bertone, G., \& Merritt, D. 2005, Phys. Rev. D, 72, \#103502

Bottino, A. et al. 2002, Phys. Rev. D, 66, \#053005

Bouquet, A., \& Salati, P. 1989, ApJ, 346, 284

CDMS Collaboration 2006, Phys. Rev. Lett., 96, \#011302

Eisenstein, D. J. et al. 2006, ApJS, 167, 40

Genzel, R. et al. 2000, MNRAS, 317, 348

Ghez, A. M. et al. 2003, ApJ, 586, L127

Ghez, A. M. et al. 2005, ApJ, 620, 744

Gnedin, O. Y., \& Primack, J. R. 2004, Phys. Rev. Lett., 93, \#061302

Gondolo, P., \& Silk, J. 1999, Phys. Rev. Lett., 83, 1719

Gould, A. 1987, ApJ, 321, 571

Greenstein, J. L., \& Trimble, V. L. 1967, ApJ, 149, 283

Griest, K., \& Seckel, D. 1987, Nucl. Phys. B, 296, 681

Haber, H. E., \& Kane, G. L. 1985, Phys. Rep., 117, 75
Hamada, T., \& Salpeter, E. E. 1961, ApJ, 134, 683

Hansen, B. 2004, Phys. Rep., 399, 1

Jungman, G., Kamionkowski, M.,\& Griest, K. 1996, Phys. Rep., 267, 195

Moore, B., Quinn, T., Governato, F., Stadel, J., \& Lake, G. 1999, MNRAS, 310, 1147

Moskalenko, I. V., \& Wai, L. L., 2006, arXiv: astro-ph/0608535

Navarro, J. F., Frenk, C. S., \& White, S. D. M. 1997, ApJ, 490, 493

Panei, J. A., Althaus, L. G., \& Benvenuto, O. G. 2000, A\&A, 353, 970

Rieke, G. H., Rieke, M. J. \& Paul, A. E. 1989, ApJ, 336, 752

Salati, P., \& Silk, J. 1989, ApJ, 338, 24

Ullio, P., Zhao, H. S., \& Kamionkowski, M. 2001, Phys. Rev. D, 64, \#043504

Werner, K., \& Wolff, B. 1999, A\&A, 347, L9 